\title{
A Comparison of Bone Resorption Over Time : An Analysis of the Free Scapular, lliac Crest, and Fibular Microvascular Flaps in Mandibular Reconstruction
}

\section{Wilkman, Tommy}

2017-03

Wilkman , T , Apajalahti , S , Wilkman , E , Törnwall , J \& Lassus , P 2017 , ' A Comparison of Bone Resorption Over Time : An Analysis of the Free Scapular, lliac Crest, and Fibular Microvascular Flaps in Mandibular Reconstruction ' , Journal of Oral and Maxillofacial Surgery , vol. 75 , no. 3 , pp. 616-621 . https://doi.org/10.1016/j.joms.2016.09.009

http://hdl.handle.net/10138/236749

https://doi.org/10.1016/j.joms.2016.09.009

publishedVersion

Downloaded from Helda, University of Helsinki institutional repository.

This is an electronic reprint of the original article.

This reprint may differ from the original in pagination and typographic detail.

Please cite the original version. 


\title{
A Comparison of Bone Resorption Over Time: An Analysis of the Free Scapular, Iliac Crest, and Fibular Microvascular Flaps in Mandibular Reconstruction
}

\author{
Tommy Wilkman, MD, DDS, ${ }^{\star}$ Satu Apajalabti, DDS, PhD, † Erika Wilkman, MD, DDS, PhD, $\ddagger$ \\ Jyrki Törnwall, MD, DDS, PhD, $\S$ and Patrik Lassus, $M D, P b D / /$
}

Purpose: The aim of the present study was to compare the resorption of the bone in the free scapular, free iliac crest, and free fibular microvascular flaps in mandibular reconstruction over time.

Patients and Methods: In the present retrospective study, we analyzed 186 consecutive patients with scapular, fibular, or deep circumflex iliac artery (DCIA) osseous free microvascular flaps in mandibular reconstruction. We followed up the patients clinically and using multislice computed tomography (MSCT) with volume analyses of the bone. The volume of the bone was analyzed against time.

Results: A total of 38 patients fulfilled the study criteria. Resorption of the osseous flaps was found to continue for several years. At 2 years, the volume loss was $14 \%$ for the scapula, 3\% for the DCIA, and $1 \%$ for the fibula. Three-dimensional (3D) volume analysis of the MSCT scans showed more resorption than 2-dimensional analyses of the radiographs. Postoperative radiation therapy, patient age, and patient gender did not correlate with bone resorption.

Conclusions: After microvascular mandibular reconstruction, the volume reduction over time is the least in the fibula and the greatest in scapula, with that of the DCIA in between. The volume reduction continues for several years in all of these. For assessment of the volume reduction of osseal reconstruction, a $3 \mathrm{D}$ volume analysis is more reliable than height by width measurements.

(C) 2016 American Association of Oral and Maxillofacial Surgeons

J Oral Maxillofac Surg 75:616-621, 2017

Composite microvascular tissue is considered the reference standard in reconstructive surgery of ablative or traumatic defects of the head and neck area. ${ }^{1-5}$ The most frequently used osteomyocutaneous free flaps are taken from the fibula, scapula, and iliac crest (deep circumflex iliac artery flap [DCIA]). ${ }^{6,7}$ Several centers use one or several flaps in their regimen. Each of the flaps has different properties, including donor morbidity, volume and quality of the osseous component, the possibility of osteotomies, the length and size of the vascular pedicle, and versatility regarding the soft tissue component. ${ }^{6,7}$

Earlier studies of bone resorption and remodeling after microvascular bone transfer mainly used measurements of panoramic radiographs. ${ }^{8-12}$ This method of imaging, however, does not allow for
Received from Helsinki University Hospital, University of Helsinki,

Helsinki, Finland.

*Senior Consultant, Departments of Plastic Surgery and Oral and Maxillofacial Surgery.

$\dagger$ Senior Consultant, Department of Radiology and HUS Medical Imaging Center.

$\ddagger$ Senior Consultant, Divisions of Intensive Care Medicine and Anaesthesiology.

$\S$ Head of Department, Department of Oral and Maxillofacial Surgery.

||Head of Department, Department of Plastic Surgery.
Conflict of Interest Disclosures: None of the authors have any relevant financial relationship(s) with a commercial interest.

Address correspondence and reprint requests to Dr Wilkman: Departments of Oral and Maxillofacial Surgery and Plastic Surgery, Helsinki University Hospital, University of Helsinki, PO Box 266, Helsinki 00029, Finland; e-mail: tommy.wilkman@hus.fi Received July 292016 Accepted September 62016

(c) 2016 American Association of Oral and Maxillofacial Surgeons 0278-2391/16/30816-3

http://dx.doi.org/10.1016/i.joms.2016.09.009 
3-dimensional (3D) analysis. Previously, assessments have been performed using measurements of the height or width of the flap but not the actual volume. In general, these studies have only reported minor resorption of the osseous parts. The resorption is fastest during the first 6 to 12 months and seems to decline later; however, evidence exists that lesser changes might occur later on. ${ }^{13}$ Remodeling of the bone can alter the biomechanical properties of the bone and can also cause hypertrophy of fibular grafts. ${ }^{13}$ Pure resorption of the bone volume can be considered a complication caused by insufficient blood flow due to flap properties, the use of osteotomies, or installation of dental implants.

Because we routinely use microvascular scapula, DCIA, and fibula flaps with mandibular reconstruction in our unit, we decided to compare the resorption of the bone volume over time for these 3 different flaps. The study was performed using multislice computed tomography (MSCT) scans with volume analyses.

\section{Patients and Methods}

The research ethics board of the Helsinki University Hospital approved the present study on May 11, 2011. In our retrospective study, we analyzed 186 consecutive patients with head and neck cancer who had undergone reconstruction with osseous free microvascular flaps from 2001 to 2013 at the Departments of Plastic Surgery and Maxillofacial Surgery at Helsinki University Hospital, University of Helsinki (Helsinki, Finland). All the patients were assessed pre- and postoperatively by the weekly multidisciplinary head and neck cancer board. Of the 186 patients, 136 had undergone mandibular, 44 maxillary, and 7 orbital reconstructions. We chose to include only the mandibular reconstructions in the present study to ensure the coherence of the study population. The mandibular reconstructions included 73 free iliac crest (DCIA) flaps, 34 scapular flaps, and 23 fibular flaps. Six radial and metatarsal bone flaps were excluded. The DCIA, scapula, and fibula flaps were the aim of the present study.

\section{STANDARDIZED MSCT SCANS}

We followed up the patients clinically and with MSCT imaging studies. Our protocol included MSCT or magnetic resonance imaging (MRI) at 6,12 , and 24 months or when clinically indicated. We assessed the volume of the bone flaps initially using standardized MSCT and as a part of the normal follow-up protocol, using the Advantage Workstation software, version 4.4 (GE Healthcare, Port Washington, NY). The CT examination was performed using highresolution helical CT scanning with a bone algorithm.
Volume measurements were performed on 1.0-, 2.0-, or $2.5-\mathrm{mm}$-thick reformatted axial images. Volume analysis of the bone is possible using CT data if scanning is performed with a straight tube without tilt of gantry. A total of 21 patients fulfilled these primary criteria.

\section{BONE THICKNESS AND HEIGHT}

In addition to the volume analysis, we measured the bone height and thickness in millimeters from each segment of microvascular bone transfer at a distance of $10 \mathrm{~mm}$ from the osteotomy to exclude errors caused by callus formation or osteolysis of the osteotomy. In flaps with 1 or more osteotomies, each segment was analyzed. At least 2 postoperative CT scans were needed. Seventeen patients had undergone at least 1 postoperative CT scan with a different protocol; thus, we excluded them from the volume analysis. In these 17 patients, the bone stock was measured using the height and thickness only. Patients followed up using MRI or pantomography were excluded from the present analysis.

Thus, 38 patients, 22 men and 16 women, were included in the present study. Of the 38 patients, 2 had a benign odontogenic tumor (ameloblastoma), 1, a vascular malformation, and 35 , malignant disease. Of the 35 patients, 1 had an osteosarcoma, 1 an ameloblastic carcinoma, 1 had a basosquamous carcinoma, and 32 had squamous cell carcinoma. All but 7 patients with malignant disease had undergone postoperative radiotherapy. None of the patients with benign lesions had undergone radiotherapy.

The volume analysis was double blinded and done twice per case by 2 of us (T.W.; S.A. [maxillofacial radiologist]) to secure the results by intra- and interinvestigator correlation. One of us (S.A.) performed the height by thickness measurements.

To enable the comparative volume analysis of the 3 different bone flaps over time, but with the CT examinations performed at nonstandardized intervals, the first postoperative volume was defined as 1.00 and the following measurements as a relative volume reduction of the first volume measured. The crosssection measurements of height and thickness of the bone flap were calculated as millimeters $\times$ millimeters, giving a relative area of the cross-section. This was also formatted as a relative value of 1.00 on the first postoperative CT scan. The volume and crosssection analyses were done separately.

For statistical analysis and the estimation of the decrease of bone volume in the different bone flaps over time, we used simple linear curve fitting (NCSS, version 8; NCSS Statistical Software, East Kaysville, UT). We analyzed the data using SPSS, version 20 (IBM Corp, Armonk, NY). We used Pearson's 
correlation coefficient to validate the measurements and assess the correlations of the continuous variables. We used the Mann-Whitney $U$ test for assessments to compare groups of continuous data and the $\chi^{2}$ or Fisher exact test for categorical data. The results are presented as median values with the interquartile range (IQR).

\section{Results}

A total of 38 patients, 22 men and 16 women, were enrolled in the present study, and 749 measurements were taken in the analysis of 5 scapular flaps, 25 DCIA flaps, and 8 fibular flaps. The mean patient age was 61 years (range, 21 to 87). We followed up the patients with CT imaging for 7 to 132 months (median, 24.5; IQR, 17.0 to 55.5).

In the first postoperative volume analysis, the mean volume of the DCIA, scapula, and fibula was 23.8 , 11.4 , and $7.2 \mathrm{~cm}^{3}$, respectively. The interinvestigator reliability of the volume analysis was 0.997 (Pearson correlation coefficient, $P<.001)$ and the intrainvestigator reliability was 0.999 (S.A.) and 0.998 (T.W.; $P<.001$ ).

The first comparison was between the bone flaps with CT data available at 6,12 , and 24 months ( \pm 2 months; $n=20$ ). The results of the volume analysis alone showed that the reduction in the volume was $7 \%$ for the scapula, $2 \%$ for the DCIA, and $0 \%$ for the fibular flap during the first year. At 2 years, the volume loss was $14 \%$ for the scapula, $3 \%$ for the DCIA, and $1 \%$ for the fibula (Fig 1).

The second comparison included all available data $(\mathrm{n}=38)$ with linear volume analysis and height by width calculations for all bone flaps. In the follow-up examination at 48 months, the volume analysis showed a 0.69 remaining relative volume for the scapula (95\% confidence interval [CI], 0.48 to 0.90 ), 0.88 for the DCIA (95\% CI, 0.67 to 1.08 ), and 0.95 for the fibula ( $95 \%$ CI, 0.77 to 1.05 ). The height by width analysis showed a remaining relative volume of 0.89 for the scapula (95\% CI, 0.75 to 1.03 ), 0.89 for the DCIA (95\% CI, 0.77 to 1.01 ), and 0.96 for the fibula (95\% CI, 0.86 to 1.05; Figs 2 and 3, Table 1).

Postoperative radiation therapy was given to 24 of the 38 patients, and no statistical correlation with volume reduction occurred during the first 2 years. The volume reduction for all flaps was $11 \%$ in the radiated patients and $9 \%$ in the nonradiated patients (Mann-Whitney $U$ test, $P=.44$ ); 0.89 (95\% CI, 0.86 to 0.969$)$ compared with 0.91 (95\% CI, 0.89 to 1.02 ). For the radiated bone flaps, the reduction of bone volume at 2 years was $12 \%$ in the scapula $(0.88 ; 95 \% \mathrm{CI}$ 0.7 to 0.88$), 7 \%$ in the DCIA $(0.93 ; 95 \%$ CI 0.88 to $0.96)$, and $5 \%$ in the fibula $(0.95 ; 95 \% \mathrm{CI}, 1.06$ to 1.02). No statistically significant correlations were

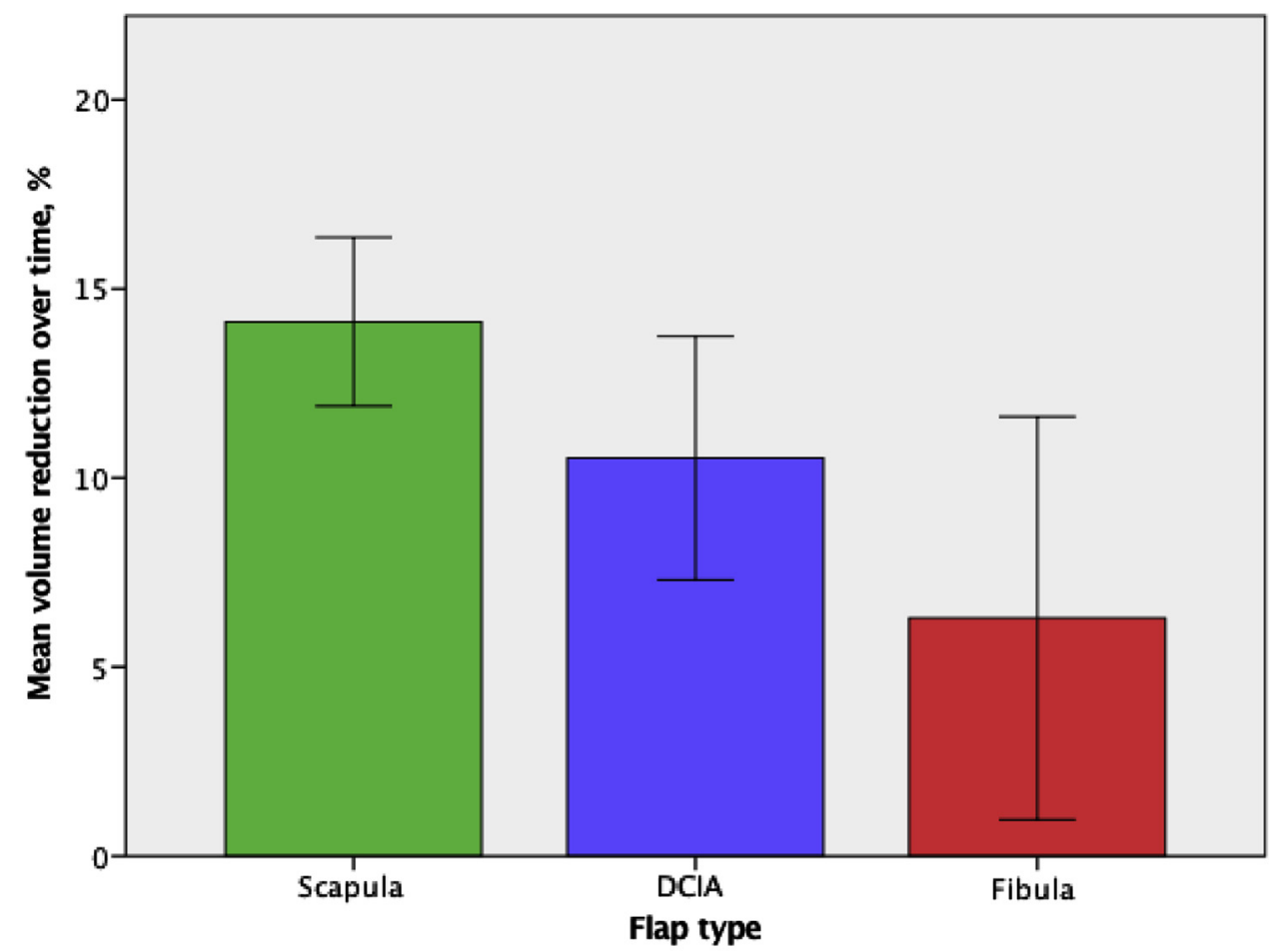

Error Bars: $95 \% \mathrm{Cl}$

FIGURE 1. Mean volume reduction at 48 months with $95 \%$ confidence interval (CI). DCIA, deep circumflex iliac artery. 


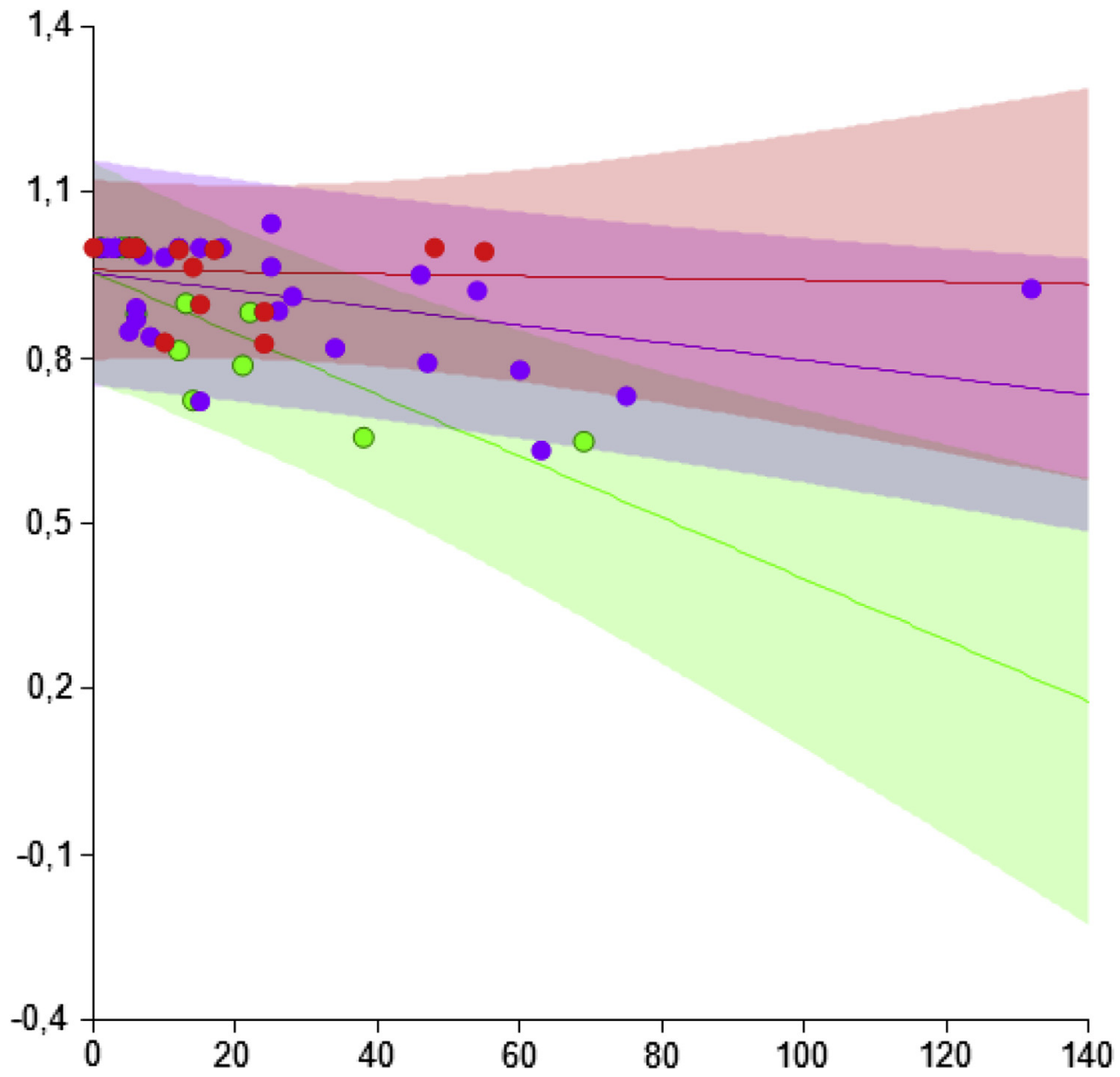

FIGURE 2. Three-dimensional volume analysis of the bone flaps showing resorption over time. Volume was related to the initial (first volume $=1.00$ ) volume of each flap. Time in months. Red line, fibula; blue line, deep circumflex iliac artery; and green line, scapula; shaded area, 95\% confidence interval.

Wilkman et al. Volume Analysis of Free Osseal Reconstructive Flaps. J Oral Maxillofac Surg 2017.

found between bone resorption and patient age $(P=.46)$ or gender $(P=.50)$. Dental implants were installed in 16 patients, 14 in the DCIA group, 2 in the fibula group, and none in the scapula group. Of the 16 patients with dental implants installed, 4 lost 1 implant each, all in the DCIA group. No losses occurred in the 2 fibular cases.

\section{Discussion}

Our data show that differences exist in the resorption over time of 3 microvascular bone flaps used in mandibular reconstruction. The fibular flap was the most stable, with $95 \%$ (95\% CI, 0.77 to $1.13 \%$ ) remaining after 48 months. In contrast, the scapular flap had the greatest loss of volume, with $69 \%$ (95\% CI, 0.48 to $0.90 \%$ ) remaining at 48 months. The DCIA had a remaining volume of $88 \%$ (95\% CI, 0.67 to $1.08 \%$ ) at 48 months. This is a new finding that could affect the long-term stability and function of the reconstruction. The fibula was the most durable, with little change in volume. The DCIA was more prone to a reduction in volume; however, it had the greatest volume initially. The scapular bone experienced more resorption than did the other 2 flaps. All these flaps sustained a substantially greater volume than nonvascularized grafts for which a $50 \%$ resorption rate can be expected after only 6 months ${ }^{14}$

Our results showed that remodeling or resorption of the ostial transplants continues for several years, not only during the first year. This has also been reported by Disa et al, ${ }^{9}$ with fibular grafts shown to resorb by 0 to $6 \%$, depending on the site, within the first 52 months and increasing to 5 to $8 \%$ at 104 months. Others have reported that the DCIA and fibula experience the most resorption in the initial 6 months. ${ }^{11}$ In contrast, in lower extremity reconstruction, the fibula has the capacity to undergo hypertrophy to sustain the load. ${ }^{15}$ It is possible that this also occurs in the mandible, especially in patients with functional mastication, and might explain differences in the resorption values reported in published studies. In our study, 


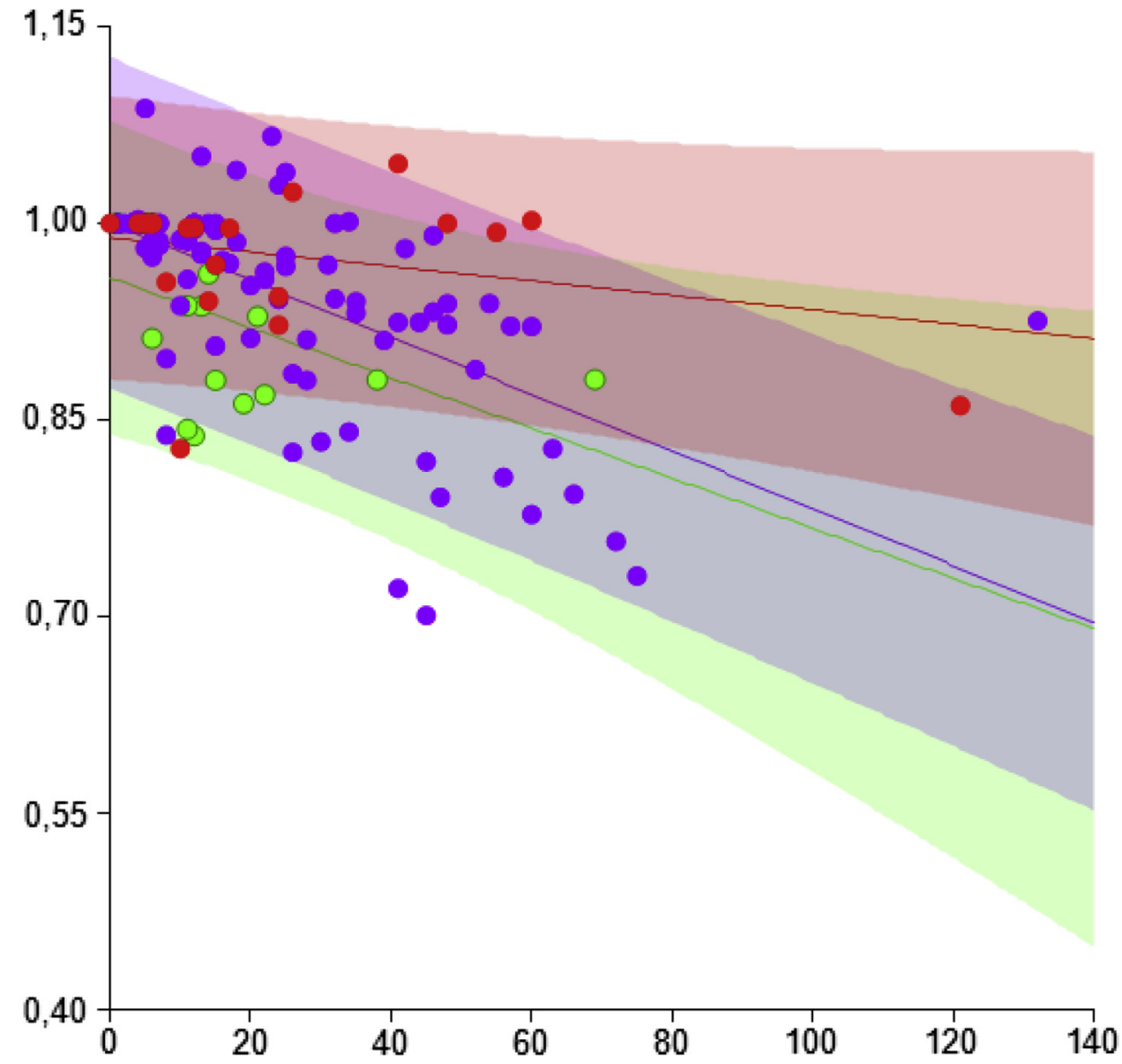

FIGURE 3. Height by width-calculated volume data for each of the bone flaps showing resorption over time. Volume related to the initial (first volume $=1.00$ ) volume of each flap. Time in months. Red line, fibula; blue line, deep circumflex iliac artery; green line, scapula; shaded area, $95 \%$ confidence interval.

Wilkman et al. Volume Analysis of Free Osseal Reconstructive Flaps. J Oral Maxillofac Surg 2017.

2 fibular grafts and 1 DCIA graft increased in volume during the follow-up period.

In our study, postoperative radiation therapy did not accelerate the resorption of the bone transplant. To our knowledge, the effect of radiation and atrophy or resorption of osseous free flaps in mandibular reconstruction has not been validated in published studies. Furthermore, the age or gender of the patient did not correlate with the results. In other studies, females have shown more atrophy of the graft but patient age seemed to be irrelevant. ${ }^{16}$

The initial volume of the bone flap is determined at surgery, with a sufficient amount of bone transferred to reconstruct the defect. The volume of the scapula flap is limited, although larger than that of the fibula; however, resorption was substantially greater than that in fibula and DCIA grafts. This is a new finding and, to the best of our knowledge, the first study to show this at a center at which all 3 of these flaps are routinely used. The natural configuration of the bone in the 3 flaps differs. The scapula is a thin bone with a thicker and quite durable lateral edge. The DCIA is a thicker block, and the fibula has a rounded triangular shape at the cross-section. This influences the measurements of the bone, especially in the height by thickness calculations previously used in published studies. ${ }^{9,10,12,17}$ The effect of the observed changes in bone volume on clinical stability and durability of the reconstruction is uncertain. All these flaps have been used with success, and dental restorations with implants are possible in the scapula with reliable results. $^{18,19}$

The comparison of the 2 methods, 3D volume analysis versus direct measurement of the height and width of the bone flaps, showed a clear difference in the results, with the simpler height by width measurement failing to record the true volume reduction, especially in the more complex shaped scapular flap. Using height by width measurements seemed more reliable for the more uniformly shaped fibula and DCIA grafts. The limitation of the present retrospective study was the low number of enrolled patients. This resulted 
from the use of several imaging modalities during the follow-up period and the varying schedules for imaging.

In conclusion, in microvascular mandibular reconstruction, the volume reduction over time will be smallest in the fibula and greatest in the scapula. We also found that the volume reduction continues for several years for all 3 of these flap types. In analyzing the volume reduction of osseous reconstruction, a $3 \mathrm{D}$ volume analysis will be more reliable than height by width measurements.

\section{References}

1. Daniel RK: Mandibular reconstruction with free tissue transfers Ann Plast Surg 1:346, 1978

2. Deschler DG, Hayden RE: Head and neck reconstruction. Neuroimag Clin North Am 6:505, 1996

3. Hurvitz KA, Kobayashi M, Evans GR: Current options in head and neck reconstruction. Plast Reconstr Surg 118:122e, 2006

4. Rinaldo A, Shaha AR, Wei WI, et al: Microvascular free flaps: A major advance in head and neck reconstruction. Acta Otolaryngol (Stockh) 122:779, 2002

5. Urken ML, Weinberg H, Vickery C, et al: Oromandibular reconstruction using microvascular composite free flaps: Report of 71 cases and a new classification scheme for bony, soft-tissue, and neurologic defects. Arch Otolaryngol Head Neck Surg $117: 733,1991$

6. Goh BT, Lee S, Tideman H, Stoelinga PJ: Mandibular reconstruction in adults: A review. Int J Oral Maxillofac Surg 37:597, 2008

7. Takushima A, Harii K, Asato H, et al: Choice of osseous and osteocutaneous flaps for mandibular reconstruction. Int J Clin Oncol 10:234, 2005

8. Benlidayi ME, Gaggl A, Buerger H, et al: Comparative study of the osseous healing process following three different techniques of bone augmentation in the mandible: An experimental study. Int J Oral Maxillofac Surg 43:1404, 2014

9. Disa JJ, Winters RM, Hidalgo DA: Long-term evaluation of bone mass in free fibula flap mandible reconstruction. Am J Surg $174: 503,1997$

10. Yla-Kotola TM, Bartlett E, Goldstein DP, et al: Union and bone resorption of free fibular flaps in mandibular reconstruction. J Reconstr Microsurg 29:427, 2013

11. Mertens C, Decker C, Engel M, et al: Early bone resorption of free microvascular reanastomized bone grafts for mandibular reconstruction-A comparison of iliac crest and fibula grafts. J Craniomaxillofac Surg 42:e217, 2014

12. Hidalgo DA, Pusic AL: Free-flap mandibular reconstruction: A 10-year follow-up study. Plast Reconstr Surg 110:438, 2002

13. Li L, Blake F, Heiland M, et al: Long-term evaluation after mandibular reconstruction with fibular grafts versus microsurgical fibular flaps. J Oral Maxillofac Surg 65:281, 2007

14. Johansson B, Grepe A, Wannfors K, Hirsch JM: A clinical study of changes in the volume of bone grafts in the atrophic maxilla. Dentomaxillofac Radiol 30:157, 2001

15. Beris AE, Lykissas MG, Korompilias AV, et al: Vascularized fibula transfer for lower limb reconstruction. Microsurgery 31:205, 2011

16. Makiguchi T, Yokoo S, Hashikawa K, et al: Evaluation of bone height of the free fibula flap in mandible reconstruction. J Craniofac Surg 26:673, 2015

17. Disa JJ, Hidalgo DA, Cordeiro PG, et al: Evaluation of bone height in osseous free flap mandible reconstruction: An indirect measure of bone mass. Plast Reconstr Surg 103:1371, 1999

18. Wilkman T, Tornwall J, Vuola J, Lassus P: The free scapular flap with latissimus muscle reduces fistulas in mandibular reconstruction. J Plast Reconstr Aesthet Surg 69:802, 2016

19. Takushima A, Harii K, Asato H, et al: Mandibular reconstruction using microvascular free flaps: A statistical analysis of 178 cases. Plast Reconstr Surg 108:1555, 2001 